\title{
Disengaged From Planning During the Lockdown? An Interview Study in an Academic Setting
}

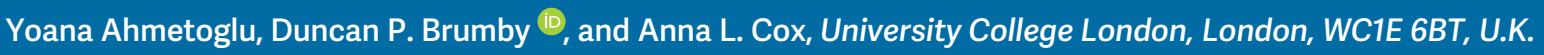

This article reports the results of a repeated interview study with 15 knowledge workers in academia during the first COVID-19 lockdown (April-July 2020). The focus of these interviews was to understand how people adjusted to working from home and the impact this had on their planning routines, strategies, and use of tools. Common themes reveal that work was chaotic, participants lost focus, and felt overworked and tired. Many disengaged from their planning routines and were reluctant to plan work in detail. However, those who reflected on how efficiently they were spending their time, found two planning strategies_breaking down tasks and manual time tracking-effective at improving accuracy of plans and productivity. Participants did not start using any new planning tools instead they adapted their existing ones. Design implications for planning tools in the context of the future of work and prevention of planning disengagement are discussed.

\section{T} HE COVID-19 PANDEMIC brought about a global crisis that suddenly required everyone, who was able to, to work entirely from home. A sudden transition to working from home (WFH) brought a set of new and unexpected challenges for these workers. One of those challenges is that workers had to quickly invent new strategies to manage and plan their work away from their normal workplace.

While work planning is a challenging task during normal times, ${ }^{1}$ it is especially so when circumstances change, such as when people change jobs, become busier, or become parents. ${ }^{2}$ Research shows that during such periods, people change their planning strategies and tools, sometimes abandoning strategies and tools altogether, and not finding new, better strategies. ${ }^{3}$ Given that job circumstances changed dramatically for millions of people during the pandemic, this period provides an unprecedented window to study the challenges knowledge workers experience in planning their work. A deep understanding of these challenges can inform the design of planning technologies that evolve with people's needs during periods of disruption and change.
The aim of this study was to explore how people adjusted to WFH and the impact this had on their planning routines. Two questions were asked. First, what kind of support do people need to advance their planning routines? Second, are there any planning strategies and tools that workers find helpful during a disruption? To answer these questions, a qualitative study was conducted, consisting of 76 weekly interviews with 15 participants during the first COVID-19 lockdown (April-July 2020). The findings show a widespread planning disengagement and emphasize the need to help workers prioritize detailed and careful planning, especially in periods of difficulties and change.

There are four contributions of this article. First, a rich description of the disruption the pandemic caused on people's work and planning routines, demonstrating a widespread planning disengagement. Second, identification of two planning strategies that were effective at improving planning and productivity. Third, providing evidence that reflection is a key driver in the development of new and better planning strategies. Fourth, design implications for planning tools in general, and for those specifically suited for remote work.
1536-1268 (C) 2021 IEEE

Digital Object Identifier 10.1109/MPRV.2021.3118900

\section{RELATED WORK}

Planning refers to decisions about which task to perform and how to prioritize them under time constraints. ${ }^{4}$ To 
plan, people use strategies which they implement through planning tools. For example, making a to-do list is a strategy that can be implemented through a paper diary or a to-do list application. The consistent implementation of those strategies through tools over time (on a daily basis, for instance) is a person's planning routine.

The effects of keeping a planning routine are beneficial. Planning prevents excessive time pressures by allowing workers to multitask between different projects, to prioritize tasks, and to ensure they have enough time to recuperate from work stress. A review of the literature concluded that planning increases feelings of being in control of one's time, productivity, job satisfaction, and health, whereas it decreases feelings of strain. 4

While planning is beneficial, it is also challenging. One of the main difficulties of planning comes from the fact that planning requires difficult time estimations. ${ }^{5}$ Research has demonstrated that people often have optimistic expectations about how quickly they can accomplish tasks. ${ }^{15}$ This is known as the planning fallacy and it has been observed in work settings. For example, a recent diary study of academics found that often only two-thirds of the work tasks planned for a given workday are achieved, resulting in significant delays to important tasks, such as research projects. ${ }^{1}$

Improving the quality of plans is important for the design of productivity systems. Experimental studies have shown that asking people to make detailed plans helps them to stay more focused compared to when vague plans are made. ${ }^{5}$ Research on productivity tools, however, focus predominantly on supporting work during the execution stage as opposed to during the planning stage of work. For instance, recent efforts include TimeToFocus, a personal informatics tool aimed at discouraging self-initiated interruptions during work, ${ }^{6}$ FlowLight, a tool that utilises Al algorithms to prevent other people from interrupting work tasks during moments of high focus, ${ }^{7}$ and research on microtasking which helps workers execute small writing tasks during unexpected moments of spare time. ${ }^{8}$

There is an increasing number of planning tools available. Examples of popular tools include Todoist (https://todoist.com) and Trello (https://trello.com). However, there is no evidence that using specialized digital planning tools helps overcome the challenges of planning. Surprisingly, planning tools have low uptake and high abandonment rates especially in academic settings. ${ }^{2,3}$ Most knowledge workers in academia still prefer to use a collection of different "self-made" tools, such as Word files, digital calendars, and notebooks. ${ }^{9}$ However, knowledge workers can get disappointed with their tools in periods when job circumstances change. Academics, for example, change their planning tools and strategies especially when disruptive events happen. ${ }^{2}$ They use planning routines, which are highly context-sensitive, and may experience difficulties in rapidly advancing them when contextual factors change (such as a sudden transition to WFH). ${ }^{1}$

In short, the COVID-19 pandemic provides an unprecedented window to study the challenges academics experience in planning their work during a significant change event. The literature suggests that the sudden shift to working entirely from home will demand more advanced and complex planning, and that people will experience difficulties in adjusting their planning routines, strategies, and tools to match these new demands. However, the reasons why they may experience these difficulties, and the support they need to advance their planning in periods of change and disruption, are yet to be understood. To investigate this we conducted a repeated interview study with 15 knowledge workers in an academic setting over the course of the first COVID-19 lockdown.

\section{METHOD}

\section{Participants}

A total of 15 participants took part in the study (see Table 1). They were academics and early career researchers at U.K. $(N=14)$ and U.S. $(N=1)$ universities (3 x lecturers, 2 x post docs, and $10 \times$ Ph.D. students). They were all WFH during data collection as a result of the COVID-19 pandemic. Participation was voluntary. The study was approved by the university ethics committee.

\section{Design and Procedure}

Lockdown in the U.K. started officially on March 23, 2020 and on different dates in March in the U.S. depending on the state. However, all participants were working remotely by 16 March. Data collection began on 6 April. Semistructured interviews were conducted each week for an average period of five weeks with each participant. Eight participants joined during the second week of April. The remaining seven participants joined gradually in the following weeks. All participants had joined the study by the beginning of June. Data collection finished in mid-July.

Interviews were conducted online through Microsoft Teams and were audio recorded. Interviews focused on how participants planned their work and whether they used new planning strategies or tools. The researcher relistened to the interview recording of each participant between each interview and noted 
TABLE 1. Summary of participants backgrounds, planning routines and tools, reported disengagement from planning, and new strategies during the study.

\begin{tabular}{|l|c|c|c|c|c|c|c|}
\hline Pp & Gender & Occupation & Household & Routine & Primary Tool & D & New Strategy \\
\hline P1 & F & Ph.D. student & Family (no children) & Weekly & Paper diary & Y & BDT \\
\hline P2 & M & Postdoc & Alone & Weekly & Paper diary & Y & None \\
\hline P3 & M & Ph.D. student & With parents & Weekly & Word & N & BDT \\
\hline P4 & M & Ph.D. student & Alone & Minimal & Notes (Mac) & Y & None \\
\hline P5 & F & Ph.D. student & With partner & Daily & Notebook & Y & BDT \\
\hline P6 & M & Ph.D. student & Family (children) & Minimal & Paper & Y & None \\
\hline P7 & F & Ph.D. student & With partner & D+W & Word & Y & MTT \\
\hline P8 & M & Ph.D. student & Alone & Minimal & Paper & N & None \\
\hline P9 & F & Ph.D. student & Family (no children) & Weekly & GoodNotes5 & N & BDT \\
\hline P10 & F & Ph.D. student & Family (children) & Minimal & Paper & Y & None \\
\hline P11 & M & Lecturer & Family (no children) & Daily & Trello & Y & BDT \\
\hline P12 & F & Lecturer & Family (children) & Minimal & Paper & Y & None \\
\hline P13 & F & Postdoc & With partner & D+W & Trello & N & MTT \\
\hline P14 & F & Ph.D. student & Alone & Daily & Paper diary & N & MTT \\
\hline P15 & F & Lecturer & Family (children) & Minimal & Word & Y & None \\
\hline
\end{tabular}

$\mathrm{Pp}=$ participant number; $\mathrm{D}=$ Disengagement from planning; $\mathrm{D}+\mathrm{W}=$ Daily and weekly; $\mathrm{BDT}=$ breaking down tasks; $\mathrm{MTT}=$ manual time tracking.

down questions to follow-up on. Recordings were transcribed with Trint (https://trint.com) and edited manually by the researcher to correct for mistakes. Data were then thematically analysed with NVIVO 12.

\section{FINDINGS}

The data consists of 76 interviews with a total duration of 16 hours 30 minutes. Each participant participated in five weekly interviews on average (range from 4 to 6 ), with a mean duration of 13 minutes.

\section{Challenges Brought by Lockdown Poor Ergonomics}

Participants were struggling with physical pain and focus issues due to poor ergonomics: The distance between my desk and bed is about a meter. Initially, I got to do 20 minutes of work and then I'd be needing to move around and stretch (P2). Having a suitable work station at home had immediate positive effects: Having a desk, monitor, mouse and keyboard has been really helping me a lot with producitivity. (P9). When participants were experiencing a drop in work focus and motivation, they often re-arranged their home office. As P8 shared: From the third weekend, I felt it was difficult to focus and I rearranged the room layout in my bedroom.

\section{Distractions by Pandemic-Related Content}

During the early stage of the lockdown, participants prioritized staying in touch with family and coworkers, and followed the news. Work focus was diminished: It was just a burnout with the emails and all the messages, and I could not get anything done because it was just so much information at the same time. I was checking the news quite a lot (P7). However, as weeks went by participants noticed that their focus and concentration was naturally improving. P4 noted that it was easier to go into a work mode in the mornings.

\section{Blurred Temporal and Spatial Boundaries}

Participants acknowledged a need to better separate their work and nonwork lives. I think I need a clear separation between work and rest [...] not only about the spatial work space but also about the temporary dimension (P8).

Commuting was no longer serving as a natural separation between work and nonwork times. When you're commuting you start getting in the mindset of working and then when you come back, you start getting the mindset of relaxing (P4).

There were no social cues to serve as reminder to stop working. It's difficult to stay focused when you don't have other people around you working. And if I 
start working, I might be working until 19:30 because other people aren't around leaving the office (P2).

Family duties were highly unpredictable. If a child starts crying, everything else has to be moved to accommodate that (P15).

Participants shared that using the same devices for work and nonwork activities was challenging. I have always connected using my laptop with nighttime, like watching Netflix (P10).

\section{Feeling Overworked and Tired}

Many participants had to change the directions of their research projects due to the new constraints of the lockdown which created extra work. We've had a lot of issues around dissertations and people not being able to collect data and having to change the research questions and the data they would collect (P15).

Participants who were living with their children experienced excessive tiredness. I do a little bit of work when the kids go to bed, but I'm just sick, exhausted that I want to go to sleep (P6).

The added challenges of lockdown fatigue, social isolation, not having rest in the weekend, all made it seem like a regular day of work during lockdown was equal to a much longer workday before the lockdown. There is nothing to look forward to in the weekend [...] I'm tired all the time (P2).

\section{Changing Expectations for Productivity}

Many participants thought that lockdown would not last long: We all thought this would last about three to four weeks and we'd go back to normal (P10). Participants continued to work at a similar pace as before. I've been working really hard during the week [...] I do the things that I used to do before everything happened (P2).

Participants noted that they had put high expectations on themselves at the start of the lockdown. Many tried to relax the self-imposed schedules they had created. I was very strict with myself waking up at 7AM and starting working by 8AM. I try to tell myself that it is fine to feel like starting work slightly later (P13).

Participants' colleagues were understanding of the challenging situation and did not expect others to maintain the same level of productivity as before. There is an informal understanding around the fact that people are not following their regular schedule. Say a student tells me "look, I've not been able to work on this," normally I might have pushed them a little bit but right now I don't push them the same way. I think all of us are working to that changed understanding (P15).

At the end of the study, participants often recognised that lowering expectations for productivity was a key approach to the pandemic. We need to cut each other a bit of slack. Being sensitive to each other's needs and what the environments will allow for and we'll get through it. We'll just have to adjust and adapt to new ways of working (P12).

\section{Planning Disengagement}

Throughout this article, participants frequently reported relying less on a planning routine. They also expressed a general reluctance to spend time on planning. Table 1 shows participants planning routines and tools used during the study, and whether they reported disengaging from planning. Minimal routines refers to using planning only when necessary, daily routines refers to making daily task lists, weekly routines refers to weekly task lists, and daily plus weekly refers to making lists once a week together with daily ones. This classification follows previous research with the same user group. ${ }^{1}$

\section{Adjustment}

One of the reasons to not engage in planing was because participants were overwhelmed when adjusting to the new situation. They felt distracted and could only focus on small urgent tasks: It was quite hard to actually focus on my tasks. If something was coming up, an email or someone texting you, that had the priority. I was not making a plan (P7).

\section{Busyness}

Participants relied less on their planning routines during extremely busy periods. Over the course of the study, many participants experienced high workloads due to extra teaching duties, or due to homeschooling. They minimized planning to scheduling activities and a short to-do list. After a busy or heavily disrupted period passed, participants reengaged with planning.

P6: There is no time to plan, it's just doing. I squeeze in bits of work when I find a moment [...] Yesterday I woke up at 5AM to try to get some work done. [four weeks later]This morning has been my first day of planning. I'm making a new timeline for my research up until the end of my Ph.D.

Participants said that careful planning took time that they lacked. There is no space anymore so I write in the margins. There's nothing organized. The [paper] has a stain of coffee. It's all messy, but if I want to get it organized, I have to write it again. That takes time. I don't want to do that (P10). Many were sceptical about the value of making detailed plans during busy periods: there's no time to think about planning and it would not work (P1). 


\section{Failing to Predict the Workday}

Lockdown schedules were inconsistent as participants were settling down into new routines. Often participants could not predict what they would want to do later on during the day which made planning more difficult. I did not plan because I did not know how I would feel. I could not predict what I would want to do (P11).

To complicate planning even more, often participants' work depended on other people. I'm basically not entirely in control of the timings. I can't anticipate when a large stream of participants will come in. You know, and with all COVID delays.

\section{Repetitive or Single Project Work}

Participants said there was less need to plan when they were doing a repetitive task or working on a single project. Having a mental plan was enough for them to remember what to do next. On days I don't update the list it is usually because I only have to work on one task (rather than two or more), which I can remember (P5).

\section{New Planning Strategies}

Even though participants disengaged from planning during some periods of the lockdown, some also started to engage in two planning strategies, which they found helpful for their productivity: breaking down tasks and manual time tracking. See Table 1 for details about the strategies used by individual participants.

\section{Breaking Down Tasks}

A planning strategy that several participants adopted during the lockdown was to break down their tasks into smaller units (than what they were already doing) while planning. In this way, they could set more achievable plans, feel better about work accomplishments, and increase their productivity.

P7 was already planning specific daily tasks but decided to make more detailed plans. This strategy meant she could learn how much time was being given to different tasks and the accuracy of her plans improved. One thing I changed recently is that I was setting even smaller tasks for each day. That really helped, it can teach you how much to allocate for tasks in the future and how much work you can get done in a day (P7).

P1 also decided to record all the small tasks in her weekly diary; previously, she did not consider small tasks to be real work but rather a distraction from getting real work done. This strategy boosted her sense of productivity as she noticed that the time she spent on the most important work tasks increased. I think I put myself like very big goals and only the small ones get done. And the big ones, I never get them. [two weeks later]Instead of planning just to write, I tried to visualize everything else I had to do. This week my planner is full of small to-do things that were as simple as emailing people or planning this meeting. Those were things that I was still doing but I was considering them things in the way of what I had to do.

\section{Manual Time Tracking}

Manual time tracking was a strategy in which participants kept a time log of their activities, often along with estimates of the time they thought a task would take to complete. Participants who tracked their time were often feeling like they were always falling behind schedule. I have estimated approximately how many hours [a task] should take because I underestimate that and I end up feeling very stressed (P14).

Time tracking also helped participants become more accurate at planning. When I plan to do something in the future, I might think it will take two hours but I can go back and see that it actually took me three days (P4).

\section{New Planning Tool Needs}

Participants did not start using any new planning tools. Instead, they changed or expressed wanting to change the way they were using their existing ones during the lockdown.

\section{Paper Tools}

During the lockdown, participants were relying more heavily on paper tools instead of digital ones. I have also a digital agenda that I'm using less because now that everything is in the same place I can't lose my todo (P7).

They felt that using paper was a more natural way of planning. We use pen and paper from school or kindergarten. It feels more familiar rather than software which always change (P5).

They also enjoyed not having to see any digital work-related content when using a notebook to plan. I like that you don't have to open the computer to plan your day (P11).

\section{Combining Work and Personal Plans}

During the lockdown, participants started to combine work and personal plans in the same planning tool. I now have personal tasks in my morning plan because I like to take longer breaks and do house tasks in the middle of the day (P3).

Sometimes this new way of planning was aimed at achieving more balanced workday. I try to combine exercise plans together with work in my todo because 
in the last two weeks I was working very efficiently but I just worked. I felt psychologically unhealthy (P8).

\section{Aligned Planning in the Household}

Planning was spread across more than one person's tool. Participants had to consider everyone else in the household when making their plans. For instance, they shared calendars to take turns in looking after children: My partner and I share calendars and we have to collaborate even more closely now and be sympathetic to each other's tasks and commitments (P12).

\section{Reflection as Driver of Change}

Participating in the study provided participants with opportunities to stop and reflect. Reflecting on how well they were managing their time was a key reason for participants to engage in new planning strategies. Talking every week was a big motivation for me because it forced me to think about [planning]. Sometimes weeks go by and then after three weeks I realize I have done nothing. This has been a good reminder of "What have I done? What active steps did it take to make my situation better instead of worse?" (P1).

The interviews reminded people of strategies they already knew would be useful: I realized that we were talking about how creating subtasks was a good thing. And I knew that from before, but I wasn't really engaging in that. [...] Ended up being pretty productive. I was very happy because the weeks before that I kept not meeting my objectives. (P7).

Some participants had more opportunities for reflection than others. Those who had additional household duties such as homeschooling or housework noted that they lacked opportunities to slow down and reflect. With everything being blended in a way, you don't have those moments where you're forced to take some time out, when you're traveling or you're walking somewhere. Right now I feel like that reflective self time I don't have (P15).

\section{DISCUSSION}

The aim of this repeated interview study was to explore how people adjusted to WFH and the impact this had on their planning routines. Consistent with previous studies conducted during the COVID-19 pandemic with various groups of knowledge workers, participants' routines and schedule were heavily disrupted. ${ }^{10,11}$ They reported negative effects on their productivity and wellbeing due to poor ergonomics, inconsistent schedule, lack of boundaries, overworking, and interruptions during work. All these factors have been previously related to psychological distress. ${ }^{10,12}$
A novel finding of the study was that participants reported that planning ahead became increasingly challenging. While work planning is instrumental for managing deadlines and time pressures, ${ }^{4}$ many participants said that they were engaging less than usual in their planning routines.

There are at least two explanations behind the observed planning disengagement. First, it can be explained with the tendency to focus on urgent tasks when under time pressure. Similar observation was reported in other work contexts, such as in email behavior. Cox et al..$^{13}$ showed that people prioritize sending responses to time-sensitive (urgent) messages over sending responses to valuable (important) messages. They explain this finding with dual-process theory of decision-making. ${ }^{14}$ When under time pressure, people rely on instinctive quick decisions (System 1) and execute the urgent tasks as opposed to rational deliberate decisions (System 2) to delay urgent responses and prioritize most important tasks first. However, when people reflect on their choices they are more likely to use System 2 , which explains why those who engaged in reflection decided to try out new planning strategies (given that planning is an important and nonurgent task) instead of only focusing on the most urgent tasks at hand and disengaging from planning.

Second, previous research shows that one of the main challenges of planning are time estimations. ${ }^{5}$ Estimating the duration of work tasks was more difficult when transitioning to remote work in lockdown because workers were not settled into a routine and incoming tasks were often delayed. Many did not see the value of attempting to plan an unpredictable workday.

Planning disengagement is likely to occur beyond the context of the lockdown during other busy and disrupted periods, such as when people transition to remote work, change jobs, become parents, return to work from leave, or get promoted. All these circumstances increase busyness and unpredictability of workdays. It is important to target those periods to provide workers with additional planning support.

Technologies in the future of work should raise users' awareness about what people lose when disengaging from planning, and help them take active steps in the formation of healthy planning habits. One way to do is to use behavior change theory to inform design of tools or add-ons to existing tools. For example, functionalities should focus on supporting reflection and habit formation, for instance, by monitoring planning habits, and by increasing the social accountability of planning. One avenue for future research, building on an approach taken by Direito et $a l .{ }^{16}$ would be to review existing planning tools on the market to find out the extent to which 
they implement behavior change techniques. By doing so, gaps in tools can be identified, and filled to better manage disengagements from planning.

Reflection is a widely used behavior change technique and has been recently recognised as a driver of positive work habits. Reflection in this study emerged organically, embedded in the weekly interview discussions. In line with this observation, a focus group study found that discussing and sharing self-tracking data facilitates opportunities for change and helps people feel motivated to try out new strategies. ${ }^{17}$ In addition, recent conversational agents have been deployed to support work detachment and reattachment through daily planning and reflection. ${ }^{18}$ Therefore, planning tools can benefit from functionalities which encourage reflection, such as conversational agents, and more ways to share planning data with others.

Apart from reflection, this study showed that two planning strategies-breaking down tasks and manual time tracking-were particularly useful for improving accuracy of plans, lowering stress, and supporting productivity. Studies have demonstrated that when people are asked to break down tasks or recall their actual previous duration, they tend to give longer and less biased estimates in their planning. ${ }^{19}$ What is surprising is that workers underestimated the usefulness of those strategies, and it was not until they engaged in them that they realized the benefits.

Furthermore, this study found that participants tracked time manually despite the abundance of automatic time tracking technology. There could be at least three reasons. First, automatic trackers do not record the same data as what participants collected. Trackers record activities, such as time in Google Scholar, whereas participants recorded their main task, such as literature review, and the latter may involve more than one activity. Second, trackers generally support reflection through visualizations of data about time wasting applications instead of data about accuracy of plans (as participants did). Third, manual tracking is more effortful than automatic tracking. It urges people to pay close attention to the data they record and, therefore, supports reflection in a better way than obtaining the data automatically. ${ }^{20}$ Those limitations of automatic tracking technologies show that they may benefit from additional functionalities, such as including to-do lists within time trackers; allowing users to play a role in time tracking in order to enrich the data about time spent in applications with data about tasks and projects; helping users reflect on the accuracy of their plans; and helping users adjust their estimates for future tasks when planning.

Finally, when working remotely, participants showed something of a "tool regression." They preferred using simpler paper tools and planned work and nonwork tasks at the same place. Given that the pandemic is likely to result in long lasting changes in working patterns and continued remote work practices, we might see a return of the paper diary, sticky notes, and notebook agendas. This point to a future challenge to support planning given a need to integrate digital tools, such as project managers and calendars, typically used by team members for collaborative planning, and physical tools for individual planning, especially in remote work context. In other words, future efforts should be directed toward better integrating and synchronising digital and nondigital planning tools, instead of making new digital planning tools that are designed to entirely replace traditional nondigital planning tools. ${ }^{9}$

\section{Limitations}

This study included knowledge workers in academia and the insights, therefore, might not generalize to all knowledge workers. That said, many of the duties and work responsibilities of academics will be shared with knowledge workers who work in different fields (e.g., responding to email, attending meetings with colleagues, completing administrative work, writing reports, etc.). Moreover, the observations reported here are likely to go beyond academics and early career researchers because what leads to planning disengagement and planning fallacies-optimism and a tendency to focus on urgent tasks-is a stable and persistent bias in human behavior. ${ }^{14,15}$

It could be argued that having participants join the study at different weeks during the first month of lockdown has confounded the insights because people were going through different stages of adjustment. However, participants were asked to provide an overview of their experience week by week before and after the pandemic started. We observed consistency across participants in terms of how they felt the first weeks impacted work and planning, which indicates that this issue should not be of significant concern.

\section{REFERENCES}

1. Y. Ahmetoglu, D. P. Brumby, and A. L. Cox, "To plan or not to plan? A mixed-methods diary study examining when, how and why knowledge work planning is inaccurate," in Proc. ACM Hum.-Comput. Interact., 2021, pp. 1-20.

2. M. Haraty, J. McGrenere, and C. Tang, "How and why personal task management behaviors change over time," in Proc. 41st Graphics Interface Conf., 2015, pp. 147-154. 
3. A. Kamsin, A. Blandford, and A. L. Cox, "Personal task management: my tools fall apart when I'm very busy!," in Proc. Extended Abstr. Hum. Factors Comput. Syst., 2012, pp. 1369-1374.

4. B. J. Claessens, W. Van Eerde, C. G. Rutte, and R. A. Roe, "A review of the time management literature," Personnel Rev., vol. 36, Feb. 13, pp. 255-276, 2007.

5. S. Koole and M. Spijker, "Overcoming the planning fallacy through willpower: Effects of implementation intentions on actual and predicted task-completion times," Eur. J. Social Psychol., vol. 30, no. 6, pp. 873-88, Nov. 2000.

6. J. Borghouts, D. P. Brumby, and A. L. Cox, "TimeToFocus: Feedback on interruption durations discourages distractions and shortens interruptions," ACM Trans. Comput.-Hum. Interact., vol. 27, no. 5, pp. 131, Aug. 17, 2020.

7. M. Züger et al., "Reducing interruptions at work: A large-scale field study of flowlight," in Proc. CHI Conf. Hum. Factors Comput. Syst., 2017, pp. 61-72.

8. S. T. Iqbal, J. Teevan, D. Liebling, and A. L. Thompson, "Multitasking with play write, a mobile microproductivity writing tool," in Proc. 31st Annu. ACM Symp. User Interface Softw. Technol., 2018, pp. 411-422.

9. A. E. Blandford and T. R. Green, "Group and individual time management tools: what you get is not what you need," Personal Ubiquitous Comput., vol. 5, no. 4, pp. 213-30, 2001.

10. A. Rudnicka, J. W. Newbold, D. Cook, M. E. Cecchinato, S. Gould, and A. L. Cox, "Eworklife: Developing effective strategies for remote working during the COVID-19 pandemic," in Proc. New Future Work Online Symp., 2020. [Online]. Available: https://www. microsoft.com/en-us/research/publication/eworklifedeveloping-effective-strategies-for-remote-workingduring-the-covid-19-pandemic/

11. J. L. Butler and S. Jaffe "Challenges and gratitude: $A$ diary study of software engineers working from home during COVID-19 pandemic," in Proc. IEEE/ACM 43rd Int. Conf. Softw. Eng., Softw. Eng. Pract., 2021, pp. 362-363.

12. G. Mark, "Multitasking in the digital age," Synth. Lectures Hum.-Centered Informat., vol. 8, no. 3, pp. 1-113, 2015.

13. A. Cox, J. Bird, D. P. Brumby, M. E. Cecchinato, and S. J. Gould, "Prioritizing unread emails: An experiment showing that people send urgent responses before important or short ones," Hum.-Comput. Interact., Nov. 25, 2020, pp. 1-24.

14. D. Kahneman, Thinking, Fast and Slow. New York, NY, USA: Farrar, Straus and Giroux, 2015.

15. D. Kahneman and A. Tversky, "Intuitive prediction: Biases and corrective procedures," Decisions and Designs Inc, Mclean, VA, USA, Tech. Rep. PTR-1042-77-6, 1977. [Online]. Available: https://citeseerx.ist.psu.edu/viewdoc/ download?doi=10.1.1.469.2095\&rep=rep1\&type=pdf
16. A. Direito, L. P. Dale, E. Shields, R. Dobson, R. Whittaker, and R. Maddison, "Do physical activity and dietary smartphone applications incorporate evidence-based behaviour change techniques?," BMC Public Health, vol. 14, no. 1, pp. 1-7, Dec. 2014.

17. R. Fleck et al., "Life-swap: How discussions around personal data can motivate desire for change," Personnel Ubiquit Comput., vol. 24, pp. 669-681, 2020.

18. A. C. Williams, H. Kaur, G. Mark, A. L. Thompson, S. T. Iqbal, and J. Teevan, "Supporting workplace detachment and reattachment with conversational intelligence," in Proc. CHI Conf. Hum. Factors Comput. Syst., 2018, pp. 1-13.

19. R. Buehler, D. Griffin, and M. Ross, "Inside the planning fallacy: The causes and consequences of optimistic time predictions," in Heuristics and Biases: The Psychology of Intuitive Judgment. Cambridge, U.K.: Cambridge Univ. Press, 2002, pp. 250-270.

20. E. K. Choe et al., "Semi-automated tracking: A balanced approach for self-monitoring applications," IEEE Pervasive Comput., vol. 16, no. 1, pp. 74-84, Jan. 5, 2017.

YOANA AHMETOGLU is currently working toward the Ph.D. degree with University College London, London, U.K., working under the supervision of Duncan P. Brumby and Anna L. Cox. Contact her at yoana.petrova.15@ucl.ac.uk.

DUNCAN P. BRUMBY is currently a professor of human-computer interaction at University College London, London, U.K., and the director of the Human-Computer Interaction M.Sc. program. His research interests include human-computer interaction and understanding how people manage digital distractions. Brumby received the Ph.D. degree from the School of Psychology, Cardiff University, Cardiff, U.K. He is the Editor-in-Chief of the International Journal of HumanComputer Studies. He is the corresponding author of this article. Contact him at d.brumby@ucl.ac.uk.

ANNA L. COX is currently a professor of human-computer interaction at University College London, London, U.K., and the vice dean (equality, diversity inclusion) with the Faculty of Brain Sciences. Her research interests include humancomputer interaction and establishing the relationships between the design of information and communications technologies (ICTs) and behavioral outcomes, and leveraging these relationships in the design of novel interfaces and systems to support people in managing their work and wellbeing. Cox received the Ph.D. degree from the University of Hertfordshire, Hatfield, U.K. Contact her at anna.cox@ucl.ac.uk. 